\title{
Simple methods for identification of radiative properties of highly-porous ceria ceramics in the range of semi-transparency
}

\author{
Leonid Dombrovsky $^{1^{*}}$ and Wojciech Lipiński ${ }^{2}$ \\ ${ }^{1}$ Joint Institute for High Temperatures, Krasnokazarmennaya 17A, NCHMT, 111116, Moscow, Russia \\ ${ }^{2}$ Research School of Engineering, The Australian National University, Canberra, ACT 2601, Australia \\ *E-mail: $\underline{\text { ldombr@yandex.ru }}$
}

Key words: radiative properties, ceramics, ceria, heat transfer, solar energy

\begin{abstract}
Advanced experimental-numerical methods for identification of spectral absorption and scattering properties of highly-porous ceria ceramics in the range of semi-transparency at room and elevated temperatures are presented. At room temperature, a period of quasi-steady oscillations of the sample surface temperature generated in response to recurrent laser heating at fixed values of the maximum and minimum temperature of the irradiated surface is measured along with the normal-hemispherical reflectance. Radiative properties are then identified using a combined heat transfer model. At elevated temperatures, an analytical solution proposed in an earlier study for zirconia ceramics is used to retrieve spectral absorption coefficient of ceria ceramics from the measured normal emittance.
\end{abstract}

\section{Introduction}

Highly-scattering porous ceramics are encountered in several engineering applications including hightemperature solar thermochemical processing. In particular, porous ceria ceramics have been investigated to realize solar-driven thermochemical cycles to split water and carbon dioxide for production of hydrogen and carbon monoxide [1-5].

Spectral radiative properties of porous ceria ceramics are critical to the analysis of thermal transportchemistry interactions, and consequently, to the design of solar thermochemical reactors. Absorption and scattering of the solar radiation as well as emission, absorption and scattering of thermal radiation by ceramics at high temperatures are all important radiative heat transfer phenomena in such analyses [6-10]. Consequently, both visible and near-infrared radiative properties of ceria ceramics at room and elevated temperatures are required for heat transfer modeling and morphology optimization of ceria ceramics for maximum solar-to-chemical energy conversion efficiency [11, 12].

Experimental studies $[13,14]$ have shown that porous ceria ceramics is characterized by very week absorption in the wavelength range of $0.6<\lambda<2 \mu \mathrm{m}$. Accurate determination of absorption coefficient in this range is difficult due to intense scattering, and consequently very low values of the sample transmittance. The resulting uncertainty in the absorption coefficient may be excessive for modeling high-temperature solar thermochemical reactors featuring ceria ceramics.

The traditional, experimental way to identify the main absorption and scattering characteristics of semi-transparent materials is based on spectral measurements of normal-hemispherical reflectance and transmittance of their samples. However, this approach is unsuitable if the transmittance is very small. A combined procedure presented in [11, 12] includes additional measurements of narrow-cone transmittance and computationally-expensive iterative Monte Carlo simulations. Theoretical 
predictions of porous ceria require the knowledge of the spectral complex index of refraction of bulk ceria and pore-level geometry. The complex index of refraction of ceria samples prepared by various methods has been reported in the literature [15, and literature cited therein]. Examples of studies of morphology effects of dispersed ceria media on their radiative and other characteristics relevant to thermochemical analyses can be found in [16-19].

An alternative method considered in [20] is based on thermal response to recurrent laser heating applied at an open boundary of a material sample. In a realistic range of the problem parameters, the period of surface temperature oscillations between definite maximum and minimum values appears to be sensitive to the value of the spectral absorption coefficient. As a result, the external monochromatic irradiation combined with the surface temperature measurements can be used to retrieve the spectral values of the absorption coefficient.

In the present study, we propose advancements in the experimental-numerical methodology for retrieval of the absorption coefficient of highly-porous ceria ceramics at room and elevated temperatures [21]. The method developed in [20] is employed in the present study to retrieve spectral radiative properties of highly-porous ceria ceramics at room temperature. The analysis is based on thermal and optical properties of ceria ceramics. It is shown that satisfactory estimates can be obtained even for very low values of the absorption coefficient in the near-infrared range. Additional measurements of normal emittance of the same sample heated uniformly from both sides by external radiation are considered to identify temperature dependence of absorption coefficient. This method was used in [22] for porous zirconia ceramics. It was assumed that scattering properties of ceramics are independent of temperature. An analytical solution for the relation between the normal emittance and absorption coefficient [23] is employed in the method presented in this study.

\section{Radiative transfer problem for collimated irradiation of a cold sample}

A one-dimensional (1-D) radiative transfer problem is considered. The external monochromatic radiation illuminates uniformly a layer of an isotropic material of uniform morphology along the normal. Following $[24,25]$ we use the transport approximation for the scattering phase function to simplify the scalar radiative transfer equation (RTE). After integration over an azimuthal angle, the RTE and the boundary conditions for the optically thick layer of a medium can be written as follows (subscript $\lambda$ is hereafter omitted for brevity) [24]:

$$
\begin{gathered}
\mu \frac{\partial \bar{I}(z, \mu)}{\partial z}+\beta_{\text {tr }} \bar{I}(z, \mu)=\frac{\sigma_{\operatorname{tr}}}{2} \int_{-1}^{1} \bar{I}(z, \mu) \mathrm{d} \mu \quad \mu=\cos \theta \quad z>0 \\
\bar{I}(0, \mu)=\delta(1-\mu) \quad \bar{I}(\infty,-\mu)=0 \quad \mu>0
\end{gathered}
$$

where $\bar{I}(z, \mu)=I(z, \mu) q_{\mathrm{e}}$ is the normalized (per unit incident radiative flux) spectral radiation intensity at point $\vec{r}$ in direction $\mu, \sigma_{\mathrm{tr}}=\sigma(1-\bar{\mu})$ is the transport scattering coefficient ( $\sigma$ is the ordinary scattering coefficient, $\bar{\mu}$ is the asymmetry factor of scattering), $\beta_{\mathrm{tr}}=\alpha+\sigma_{\mathrm{tr}}$ is the transport extinction coefficient, $\alpha$ is the absorption coefficient. Note that transport approximation has been widely used in radiative transfer calculations. It was confirmed that hemispherical characteristics of the radiation field in scattering materials with multiple scattering present are well described using this approximation. The first of boundary conditions (2) is written for the simplest case of a non-refracting medium. It is correct for highly-porous materials $[11,12]$. The second of conditions (2) is correct for optically thick layers when an actual geometrical thickness makes no difference for the radiative transfer problem. 
Following the usual technique [24, 26, 27], consider the radiation intensity $\bar{I}$ as a sum of the diffuse component $\bar{J}$ and collimated component:

$$
\bar{I}(z, \mu)=\bar{J}(z, \mu)+E_{\text {tr }}(z) \delta(1-\mu) \quad E_{\text {tr }}=\exp \left(-\tau_{\text {tr }}\right) \quad \tau_{\text {tr }}=\beta_{\text {tr }} z
$$

The mathematical problem statement for the diffuse component of radiation intensity is as follows:

$$
\mu \frac{\partial \bar{J}}{\partial z}+\beta_{\text {tr }} \bar{J}=\frac{\sigma_{\mathrm{tr}}}{2}\left(G+E_{\mathrm{tr}}\right) \quad G=\int_{-1}^{1} \bar{J} \mathrm{~d} \mu \quad \bar{J}(0, \mu)=\bar{J}(\infty,-\mu)=0 \quad \mu>0
$$

The normalized spectral radiation power absorbed in the medium is expressed as:

$$
\bar{W}(z)=-\int_{-1}^{1} \frac{\partial \bar{I}}{\partial z} \mu d \mu=\alpha\left(G+E_{\mathrm{tr}}\right)
$$

The problem (4) for the diffuse component of the radiation intensity is still very complex. To simplify this problem, one can employ analytical representations of the angular dependence of radiation intensity, leading to the so-called differential approximations [24, 25]. According to [28-30], the modified two-flux approximation can be used to obtain a sufficiently accurate solution for the diffuse component of the radiation field in a refracting medium. In the case of a non-refracting medium (or highly-porous ceramics), this approach coincides with the two-flux method [24]. Integrating equation (4) separately over the intervals $-1<\mu<0$ and $0<\mu<1$, one obtains the following boundary-value problem for $G(z)$ :

$$
-D G^{\prime \prime}+\alpha G=\sigma_{\mathrm{tr}} E_{\mathrm{tr}} \quad D=1 /\left(4 \beta_{\mathrm{tr}}\right) \quad D G^{\prime}(0)=G(0) / 2 \quad G^{\prime}(\infty)=0
$$

For $0.75<\omega_{\mathrm{tr}}=\sigma_{\mathrm{tr}} / \beta_{\mathrm{tr}}<1$, the problem (6) has the analytical solution:

$$
G=\frac{4}{4-3 / \omega_{\text {tr }}}\left[\frac{3}{2+\xi} \exp \left(-\xi \tau_{\text {tr }}\right)-\exp \left(-\tau_{\text {tr }}\right)\right] \quad \xi=2 \sqrt{1-\omega_{\text {tr }}}
$$

where $\omega_{\mathrm{tr}}$ in the transport scattering albedo of the medium. The resulting profile of the normalized absorbed radiation power can be written as follows:

$$
\bar{W}\left(\tau_{\text {tr }}\right)=\frac{12 \alpha}{4-3 / \omega_{\text {tr }}}\left[\frac{1}{2+\xi} \exp \left(-\xi \tau_{\text {tr }}\right)-\frac{1}{4 \omega_{\text {tr }}} \exp \left(-\tau_{\text {tr }}\right)\right]
$$

The profiles of the dimensionless quantity $\bar{W}\left(\tau_{\mathrm{tr}}\right) / \alpha$ calculated using Eq. (8) for selected $\omega_{\mathrm{tr}}$ are presented in Fig. 1. Considerable changes of the absorption profile are observed for the values of the medium scattering albedo $\omega_{\text {tr }}>0.9$, typical of weakly absorbing materials. This effect is accompanied by appearance of the absorption maximum at a distance from the irradiated surface. 


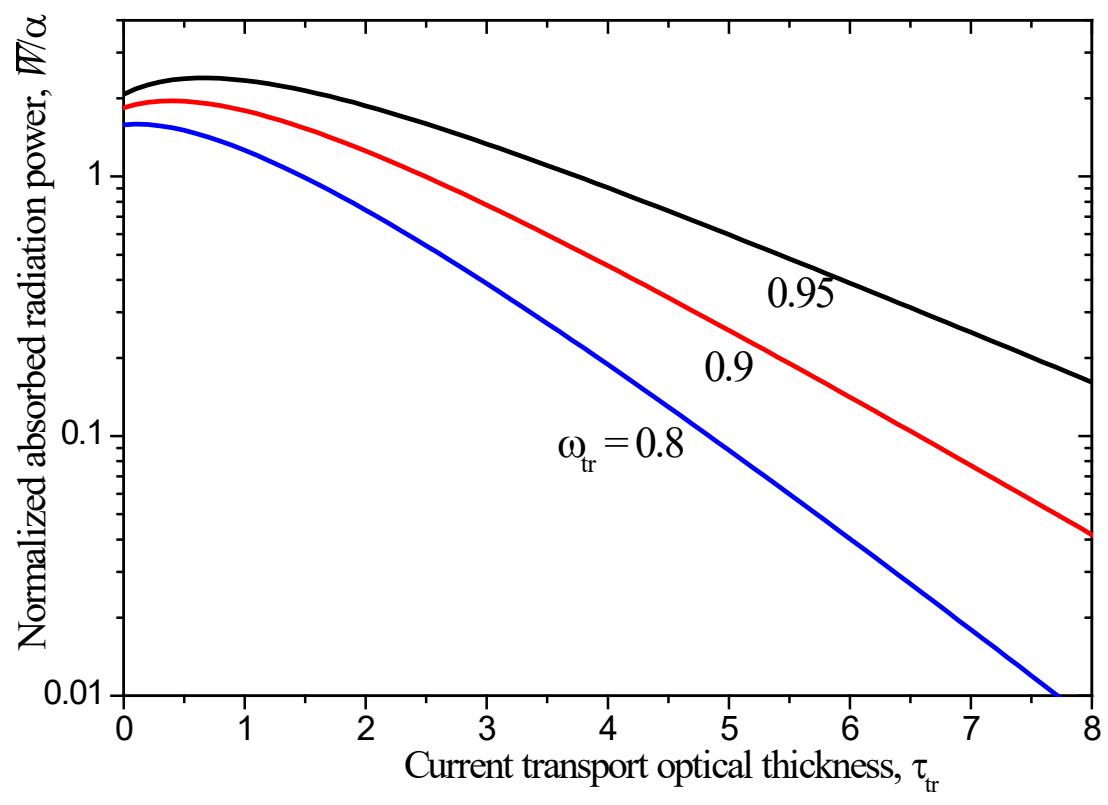

Figure 1: Typical profiles of normalized absorbed radiation power in a planar layer of a weakly absorbing medium.

According to [29], the normal-hemispherical reflectance of an optically thick non-refracting medium is determined by the medium transport albedo:

$$
R_{\mathrm{n}-\mathrm{h}}\left(\omega_{\mathrm{tr}}\right)=\frac{\omega_{\mathrm{tr}}}{1-\omega_{\mathrm{tr}}} \frac{\xi^{2} / 2}{(1+\xi)(2+\xi)}
$$

Equation (9) reduces to $R_{\mathrm{n}-\mathrm{h}}\left(\omega_{\mathrm{tr}}\right)=\omega_{\mathrm{tr}}$ in the limit of $1-\omega_{\mathrm{tr}}<<1$. So, spectral measurements of the normal-hemispherical reflectance should be made to estimate the transport albedo of the medium, whereas the values of absorption and scattering coefficients cannot be determined independently. Note that a qualitatively similar solution can be obtained for a refracting medium. The mathematical derivations leading to an analytical solution for the general case of a refractive medium with an arbitrary but constant index of refraction can be found in [31].

\section{Transient heat transfer problem for the sample of ceramics}

For monochromatic irradiation, the transient heat transfer problem in a sample of semi-transparent material is formulated as the following parabolic 1-D radiative-conductive problem:

$$
\begin{gathered}
\rho c \frac{\partial T}{\partial t}=\frac{\partial}{\partial z}\left(k \frac{\partial T}{\partial z}\right)+\bar{W}(z) q_{\mathrm{e}}(t) \\
t=0 \quad T=T_{0}(z) \quad z=0 \quad k \frac{\partial T}{\partial z}=h_{1}\left(T_{\mathrm{e}, 1}-T\right) \quad z=d \quad k \frac{\partial T}{\partial z}=h_{2}\left(T-T_{\mathrm{e}, 2}\right)
\end{gathered}
$$


In the subsequent analysis, the period of radiative heating is sought such that the problem solution does not depend on the initial condition. Consider the case of a uniform initial temperature $T_{0}=$ const, symmetric and time-invariant convective boundary conditions $\left(T_{\mathrm{e}, 1}=T_{\mathrm{e}, 2}=T_{\mathrm{e}}=\right.$ const and $h_{1}=h_{2}=h=$ const $)$, and constant thermal properties of a uniform sample ( $\rho c=$ const and $k=$ const $)$. The radiation source of constant power is switched off when the surface temperature of the irradiated surface of the sample reaches the value of $T_{\mathrm{s}}=T(t, 0)=T_{\max }$ and switched on again at $T(t, z=0)=T_{\min }<T_{\max }$.

Values of the parameters used in solving the conductive-radiative problem given by Eq. (10) are specified in Table 1. The values of the sample thickness $d$ and thermophysical properties are chosen to match those of $72 \%$-porous ceria ceramics studied in papers [11, 12], either by directly extracting their values from the references (averaged $\alpha$ and $\sigma_{\text {tr }}$ ) or by further considerations as discussed below $(k, \rho c, h)$. The magnitude of the external flux $q_{\mathrm{e}}$ and the temperatures $T_{\mathrm{e}}, T_{\min }, T_{\max }$ are selected by considering feasible experimental conditions matching the requirements of the method presented here.

Table 1. Parameters of the model problem.

\begin{tabular}{cccc}
\hline Parameter & Value & Parameter & Value \\
\hline$d(\mathrm{~mm})$ & 1 & $T_{\mathrm{e}}=T_{0}(\mathrm{~K})$ & 300 \\
\hline$k\left(\mathrm{~W} \mathrm{~m}^{-1} \mathrm{~K}^{-1}\right)$ & $0.6-0.8$ & $T_{\min }(\mathrm{K})$ & 313 \\
\hline$\rho c\left(\mathrm{MJ} \mathrm{m}^{-3} \mathrm{~K}^{-1}\right)$ & 1 & $T_{\max }(\mathrm{K})$ & 315 \\
\hline$q_{\mathrm{e}}\left(\mathrm{kW} \mathrm{m}^{-2}\right)$ & 50 & $\alpha\left(\mathrm{m}^{-1}\right)$ & $5-10$ \\
\hline$h\left(\mathrm{~W} \mathrm{~m}^{-2} \mathrm{~K}^{-1}\right)$ & $16-20$ & $\sigma_{\mathrm{tr}}\left(\mathrm{mm}^{-1}\right)$ & 20 \\
\hline
\end{tabular}

The acceptable range of the heat transfer coefficient $h$ shown in Table 1 was found in the previous study [20] to minimize the effect of uncertainty in $h$ on the sample temperature in the regime of quasisteady temperature oscillations while aiming at values that can be arranged in an experiment. It is important that the effect of thermal conductivity is relatively small, especially for the above recommended range of heat transfer coefficient, thus relaxing the requirements for the accuracy of the thermal conductivity used in the present model. This result is explained by a very small thickness of the ceramics sample.

The typical time variations of the sample temperature at the irradiated surface shown in Fig. 2 indicate nearly constant-period oscillations of the temperature after the initial heating. The temperature oscillation period is an important parameter of the quasi-steady regime of the temperature variation. We consider the effect of the absorption coefficient of ceria ceramics on the average temperature oscillation period, which was obtained over longer time interval than that shown in Fig. 2. The relationship $\alpha(\Delta t)$ is presented in Fig. 3 for $h=18 \mathrm{Wm}^{-2} \mathrm{~K}^{-1}$ and two selected values of thermal conductivity. The effect of the thermal conductivity is very small in the range of $5<\alpha<7 \mathrm{~m}^{-1}$ and increases considerably at larger values of the absorption coefficient. Note that another experimental procedure, without any irradiation of the sample, can be employed in a combination with the present theoretical approach to independently obtain the effective thermal conductivity of a porous ceramics. The results of such an experimental study might be used to validate the models of paper [16]. 
The above-discussed solution enables one to find the range of the problem parameters favourable for identification of the spectral absorption coefficient of porous ceria ceramics without the knowledge of highly-accurate data for both the thermal conductivity and the heat transfer coefficients. The values of the incident radiative flux, the conditions of heat transfer with ambient air, and the sample thickness should be chosen using an optimisation procedure for the best accuracy of the resulting absorption coefficient [32]. The development of the latter procedure is beyond the scope of the present study.

The value of heat transfer coefficient at the surface of the sample can be determined using the same experimental procedure. It is sufficient to replace the sample under investigation by another sample of a non-porous opaque material with a known thermal conductivity. In this case, the heat transfer coefficient remains the only unknown parameter of the problem. It is known that heat transfer coefficient depends on the surface roughness or porosity. It should be taken into account when determining radiative properties of ceria ceramics.

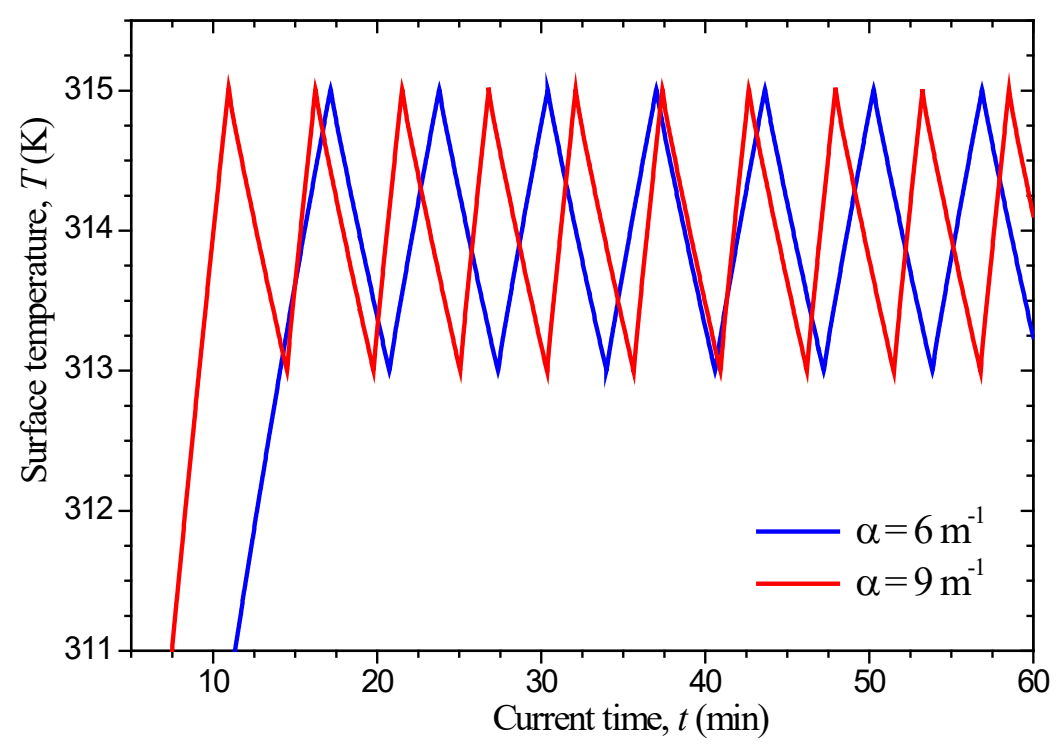

Figure 2: Time variation of irradiated-surface temperature for the ceria ceramics sample. 


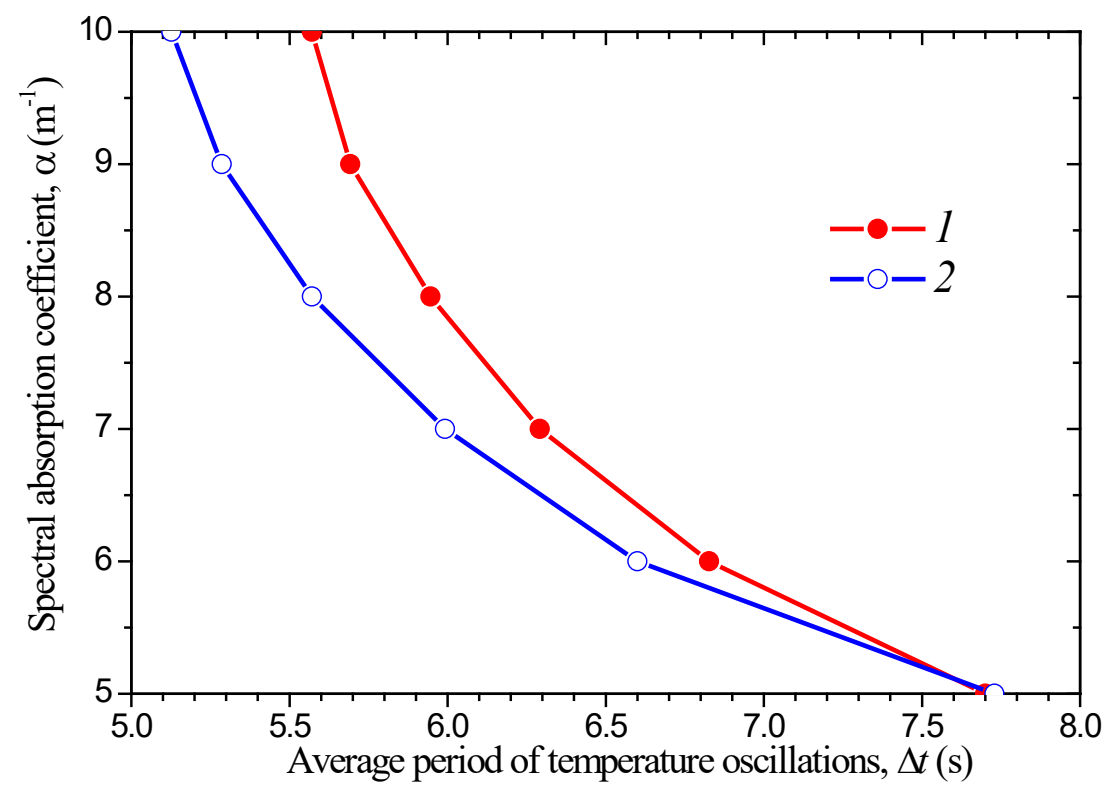

Figure 3: The predicted relation between the absorption coefficient of ceria ceramics and the average period of temperature oscillations: $1-k=0.6 \mathrm{Wm}^{-1} \mathrm{~K}^{-1}, 2-0.8 \mathrm{Wm}^{-1} \mathrm{~K}^{-1}$.

\section{The use of spectral normal emittance measurements to identify absorption coefficient at elevated temperatures}

A two-step approximate analytical solution for the spectral normal emittance of a semi-transparent sample of absorbing, scattering, and refracting medium has been derived in [23]. In the present study, the host medium is assumed to be non-refracting, and the solution can be simplified to:

$$
\varepsilon_{\mathrm{n}}=\left[1-\frac{\omega_{\mathrm{tr}}}{2 \sinh \left(\tau_{\mathrm{tr}}^{0}\right)} \frac{\frac{\sinh \left((\xi+1) \tau_{\mathrm{tr}}^{0}\right)}{\xi+1}+\frac{\sinh \left((\xi-1) \tau_{\mathrm{tr}}^{0}\right)}{\xi-1}}{\cosh \left(\xi \tau_{\mathrm{tr}}^{0}\right)+\frac{1}{2} \xi \sinh \left(\xi \tau_{\mathrm{tr}}^{0}\right)}\right]\left[1-\exp \left(-2 \tau_{\mathrm{tr}}^{0}\right)\right]
$$

where $\tau_{\mathrm{tr}}^{0}=\beta_{\mathrm{tr}} d / 2$. A direct comparison with results obtained using high-order discrete-ordinate and Monte Carlo calculations presented in [23] showed that the error of the solution (11) is sufficiently small to recommend this analytical solution for identification of the absorption coefficient at elevated temperatures.

In the limiting case of an optically thick sample $\left(\tau_{\mathrm{tr}}^{0}>>1\right)$, the expression (11) reduces to:

$$
\varepsilon_{\mathrm{n}}=1-\frac{\omega_{\mathrm{tr}}}{1+2 \sqrt{1-\omega_{\mathrm{tr}}}}
$$

For weakly-absorbing media, $1-\omega_{\mathrm{tr}}<<1$ : 


$$
\varepsilon_{\mathrm{n}}=2 \sqrt{1-\omega_{\mathrm{tr}}} \approx 2 \sqrt{\alpha / \sigma_{\mathrm{tr}}}
$$

Thus, the absorption coefficient can be readily obtained from the measured value of the normal emittance at elevated temperatures. As noted above, the scattering coefficient is practically independent of temperature. This is explained by the physical nature of scattering, which is mainly determined by the material morphology and the index of refraction, the characteristics that are weakly sensitive to the temperature of non-reacting samples [22, 33]. According to Eq. (13), the expected increase of $\varepsilon_{\mathrm{n}}$ with temperature will result in a monotonic increase of $\alpha$ with temperature, useful information for combined heat transfer calculations in the high-temperature solar thermochemical applications.

\section{Conclusions}

A recently suggested method to estimate spectral absorption coefficient of weakly-absorbing and highly-scattering media has been examined for solar thermochemical applications involving porous ceria ceramics. This method, based on thermal response of the medium to recurrent radiative heating in the spectral range of the medium semi-transparency, can be used to obtain very small absorption coefficient of ceria ceramics at room temperature in the wavelength range $0.6-2 \mu \mathrm{m}$. The required measurements of both the normal-hemispherical reflectance and the period of quasi-steady oscillations of the irradiated surface temperature of the ceramics sample between fixed values of the maximum and minimum temperatures can be readily conducted using state-of-the-art thermal laboratory equipment. Another method has been suggested for identification of the spectral absorption coefficient of highlyporous ceria ceramics at elevated temperatures. This method is based on a simple analytical relation between the measured normal emittance of an isothermal sample and the absorption coefficient. The latter measurements require advanced experimental techniques, which have already been demonstrated for other classes of high-temperature materials such as zirconia ceramics. This study has demonstrated that radiative properties of weakly absorbing and highly scattering porous ceria ceramics can be successfully determined as a function of temperature by the combined use of the two methods presented.

\section{Acknowledgements}

The financial support by the Russian Foundation for Basic Research, grant no. 16-08-00157-a (L.A. Dombrovsky), and by the Australian Research Council, grant no. FT140101213 (W. Lipiński), is gratefully acknowledged.

\section{References}

[1] S. Abanades and G. Flamant, Thermochemical hydrogen production from a two-step solar-driven water-splitting cycle based on cerium oxides, Solar Energy 80, (2006), pp. 1611-1623.

[2] W.C. Chueh and S.M. Haile, A thermochemical study of ceria: Exploiting an old material for new modes of energy conversion and $\mathrm{CO}_{2}$ mitigation, Phil. Trans. Royal Soc. A 368, (2010), pp. 32693294.

[3] W.C. Chueh, C. Falter, M. Abbott, D. Scipio, P. Furler, S.M. Haile, and A. Steinfeld, High-flux solardriven thermochemical dissociation of $\mathrm{CO}_{2}$ and $\mathrm{H}_{2} \mathrm{O}$ using nonstoichiometric ceria, Science 330, (2010), pp. 1797-1801. 
[4] J. Lapp, J.H. Davidson, and W. Lipiński, Efficiency of two-step solar thermochemical nonstoichiometric redox cycles with heat recovery, Energy 37, (2012), pp. 591-600.

[5] R. Bader, L.J. Venstrom, J.H. Davidson, and W. Lipiński, Thermodynamic analysis of isothermal redox cycling of ceria for solar fuel production, Energy \& Fuels 27, (2013), pp. 5533-5544.

[6] D. Keene, J.H. Davidson, and W. Lipiński, A model of transient heat and mass transfer in a heterogeneous medium of ceria undergoing nonstoichiometric reduction, ASME J. Heat Transfer 135, (2013), paper 052701.

[7] D. Keene, W. Lipiński, and J.H. Davidson, The effects of morphology on the thermal reduction of nonstoichiometric ceria, Chem. Eng. Sci. 111, (2014), pp. 231-243.

[8] J. Lapp and W. Lipiński, Transient three-dimensional heat transfer model of a solar thermochemical reactor for $\mathrm{H}_{2} \mathrm{O}$ and $\mathrm{CO}_{2}$ splitting via nonstoichiometric ceria redox cycling, J. Solar Energy Eng. 136, (2004), paper 031006.

[9] R. Bader, R. Bala Chandran, L.J. Venstrom, S.J. Sedler, P.T. Krenzke, R.M. De Smith, A. Banerjee, T.R. Chase, J.H. Davidson, and W. Lipiński, Design of a solar reactor to split $\mathrm{CO}_{2}$ via isothermal redox cycling of ceria, J. Solar Energy Eng. 137, (2015), paper 031007.

[10] R. Bala Chandran, R. Bader, and W. Lipiński, Transient heat and mass transfer analysis in a porous ceria structure of a novel solar redox reactor, Int. J. Thermal Sci. 92, (2015), pp. 138-149.

[11] L.A. Dombrovsky, K. Ganesan, and W. Lipiński, Combined two-flux approximation and Monte Carlo model for identification of radiative properties of highly scattering dispersed materials, Comput. Therm. Sci. 4, (2012), pp. 365-378.

[12] K. Ganesan, L.A. Dombrovsky, and W. Lipiński, Visible and near-infrared optical properties of ceria ceramics, Infrared Phys. Tech., 57, (2013), pp. 101-109.

[13] Z. Liang, W.C. Chueh, K. Ganesan, S.M. Haile, and W. Lipiński, Experimental determination of transmittance of porous cerium dioxide media in the spectral range 300-1,100 nm, Exper. Heat Transfer 24, (2011), pp. 285-299.

[14] K. Ganesan and W. Lipiński, Experimental determination of spectral transmittance of porous cerium dioxide in the range 900-1,700 nm, ASME J. Heat Transfer 133, (2011), paper 104501.

[15] K. Ganesan, L.A. Dombrovsky, T.S. Oh, and W. Lipiński, Determination of optical constants of ceria by combined analytical and experimental approaches, JOM 65, (2013), pp. 1694-1701.

[16] S. Haussener S and A. Steinfeld, Effective heat and mass transport properties of anisotropic porous ceria for solar thermochemical fuel generation, Materials, 5, (2012), pp. 192-209.

[17] L. Venstrom, N. Petkovich, S. Rudisill, A. Stein A, and J. Davidson, The effects of morphology on the oxidation of ceria by water and carbon dioxide, J. Sol. Energy Eng., 134, (2012), paper 011005 .

[18] K. Ganesan, J. Randrianalisoa, and W. Lipiński, Effect of morphology on spectral radiative properties of three-dimensionally ordered macroporous ceria packed bed, ASME J. Heat Transfer 135, (2013), paper 122701.

[19] J. Randrianalisoa and W. Lipiński, Effect of pore-level geometry on far-field radiative properties of three-dimensionally ordered macro-porous ceria particle, Appl. Optics 53, (2014), pp. 12901297.

[20] L.A. Dombrovsky, A new method to retrieve spectral absorption coefficient of highly-scattering and weakly-absorbing materials, J. Quant. Spectr. Radiat. Transfer, 172, (2016), pp. 75-82. 
[21] L.A. Dombrovsky and W. Lipiński, On retrieval of spectral radiative properties of highly-porous ceria ceramics in the range of semi-transparency, in A.J. Nowak, J. Banaszek, and B. Šarler, editors, Proceedings of the Eurotherm Seminar 109/Numerical Heat Transfer 2015, GliwiceWarsaw, Poland, 27-30 September 2015.

[22] L.A. Dombrovsky, B. Rousseau, P. Echegut, J.H. Randrianalisoa, and D. Baillis, High temperature infrared properties of YSZ electrolyte ceramics for SOFCs: Experimental determination and theoretical modeling, J. Amer. Ceram. Soc., 94, (2011), pp. 4310-4316.

[23] L.A. Dombrovsky, J.H. Randrianalisoa, W. Lipiński, and D. Baillis, Approximate analytical solution to normal emittance of semi-transparent layer of an absorbing, scattering, and refracting medium, J. Quant. Spectr. Radiat. Transfer, 112, (2011), pp. 1987-1999.

[24] L.A. Dombrovsky and D. Baillis, Thermal Radiation in Disperse Systems: An Engineering Approach, Begell House, New York, 2010.

[25] L.A. Dombrovsky, The use of transport approximation and diffusion-based models in radiative transfer calculations, Comput. Therm. Sci., 4, (2012), pp. 297-315.

[26] J.R. Howell, R. Siegel, and M.P. Mengüç, Thermal Radiation Heat Transfer, CRC Press, New York, 2010.

[27] M.F. Modest, Radiative Heat Transfer, Third edition, Academic Press, New York, 2013.

[28] L. Dombrovsky, J. Randrianalisoa, D. Baillis, and L. Pilon, Use of Mie theory to analyze experimental data to identify infrared properties of fused quartz containing bubbles, Appl. Optics 44, (2005), pp. 7021-7031.

[29] L. Dombrovsky, J. Randrianalisoa, and D. Baillis, Modified two-flux approximation for identification of radiative properties of absorbing and scattering media from directionalhemispherical measurements, J. Opt. Soc. Am. A, 23, (2006), pp. 91-98.

[30] L.A. Dombrovsky, J.H. Randrianalisoa, W. Lipiński, and V. Timchenko, Simplified approaches to radiative transfer simulations in laser induced hyperthermia of superficial tumors, Comput. Therm. Sci., 5, (2013), pp. 521-530.

[31] L.A. Dombrovsky, V. Timchenko, C. Pathak, H. Piazena, W. Müller, and M. Jackson, Radiative heating of superficial human tissues with the use of water-filtered infrared-A radiation: $A$ computational modeling, Int. J. Heat Mass Transfer, 85, (2015), pp. 311-320.

[32] T.D. Fadale, A.V. Nenarokomov, and A.F. Emery, Uncertainties in parameter estimation: the inverse problem, Int. J. Heat Mass Transfer, 38, (1995), pp. 511-518.

[33] L.A. Dombrovsky, D. Baillis, and J.H. Randrianalisoa, Some physical models used to identify and analyze infrared radiative properties of semi-transparent dispersed materials, J. Spectr. Dynam., 1, (2011), paper 7. 